Revue d'histoire de l'enfance « irrégulière »

Le Temps de l'histoire

5 | 2003

Pratiques éducatives et systèmes judiciaires

\title{
Modèles, systèmes et méthodes dans l'éducation correctionnelle en Suisse romande, 1820-1914
}

Martine Ruchat

\section{(2) OpenEdition \\ Journals}

Édition électronique

URL : http://journals.openedition.org/rhei/863

DOI : 10.4000/rhei.863

ISBN : 978-2-7535-1643-4

ISSN : $1777-540 \mathrm{X}$

Éditeur

Presses universitaires de Rennes

Édition imprimée

Date de publication : 15 novembre 2003

Pagination : 15-26

ISSN : 1287-2431

Référence électronique

Martine Ruchat, « Modèles, systèmes et méthodes dans l'éducation correctionnelle en Suisse

romande, 1820-1914», Revue d'histoire de l'enfance « irrégulière » [En ligne], 5 | 2003, mis en ligne le 02

juin 2007, consulté le 04 décembre 2020. URL : http://journals.openedition.org/rhei/863 ; DOI : https:// doi.org/10.4000/rhei.863

(c) PUR 


\section{Modèles, systèmes et méthodes dans l'éducation correctionnelle en Suisse romande, 1820-1914}

\section{Pour une histoire de l'éducation spécialisée en Suisse ${ }^{(2)}$}

La formation de la Confédération suisse, tout au long de l'Ancien Régime, à partir d'États-cantons jaloux de leur identité, de leurs particularismes communaux, religieux, culturels, économiques, n'aboutit, à la révolution radicale de 1848 , qu'à une unification relative. Cette extrême diversité politique ne rend que plus difficile le travail des historiens et historiennes de l'éducation. Concernant l'école, ceux-ci tentent aujourd'hui de constituer une histoire comparée en Suisse, ${ }^{(3)}$ travail d'envergure tant la diversité linguistique, culturelle, économique et même législative de la Suisse est grande. L'histoire sociale, l'histoire des idées, l'histoire institutionnelle, l'histoire-problème ${ }^{(4)}$ restent, sur le plan national, encore à écrire. Ni la prison, ni l'école, ni les maisons de correction n'ont aujourd'hui une histoire générale suisse.

Même cantonale, l'histoire des maisons de correction, est aussi à développer. Les travaux pionniers de Peter Chmelik, Armenerziebung- und Rettungsanstalten, ${ }^{(5)}$ datant de 1978, et ceux que nous avons effectués dès 1990, dont rendent compte L'oiseau et le cachot et Les chroniques $d u$ mal, ${ }^{(6)}$ n'ont guère suscité d'autres développements. Quelques monographies, telle celle de Pierre Avvanzino sur l'école Pestalozzi d'Echichens ${ }^{(7)}$ ou sous la forme de travaux de mémoires en histoire ${ }^{(8)}$ les complètent, sans toutefois qu'il y ait, jusqu'à aujourd'hui, un souci de synthèse.

Nonobstant cet état de l'historiographie de l'éducation spécialisée en Suisse, l'histoire helvétique de la prise en charge des enfants "en situation non-ordinaire", que ce soit en prison, dans les instituts pour crétins, sourds et aveugles, dans les asiles pour enfants abandonnés moralement, dans les maisons de correction et les colonies agricoles pour enfants vicieux et cou-
Martine

Ruchat $^{(1)}$
(1) Professeure, université de Neufchâtel.

(2) Voir Maurice Capul, «Vers une histoire de l'éducation spécialisée ", Les dossiers de l'éducation, $\mathrm{n}^{\circ} 8$ et 10, Toulouse, université de Toulouse, 1985, 1986, p. 73-94.

(3) Rita Hofstetter, Charles Magnin, Lucien Criblez, Carlo Jentzer, Une école pour la démocratie. Naissance et développement de l'école primaire publique en Suisse au 19ème siècle, Berne, Peter Lang, collection Exploration, 1999, $376 \mathrm{p}$.

(4) Antoine Léon, L'histoire de l'éducation

Martine Ruchat / p. 15 à 26 
aujourd'bui, Paris,

Unesco, Lausanne,

Delaschaux \& Niestlé, 1984.

(5) Peter Chmelik, Armenerziehung- und Rettungsanstalten, Zürich, Selbstverlag des Verfassers, 1978.

(6) Martine Ruchat, L'oiseau et le cachot. Naissance de l'éducation correctionnelle en Suisse romande, 1800-1913, ZOE, 1993, 252 p. ; Martine Ruchat, Les chroniques du mal. Le journal de l'éducation correctionnelle, Genève, Passé présent, 1998, $214 \mathrm{p}$.

(7) Pierre Avvanzino, "Histoire de l'éducation spécialisée (1827-1979). Les arcanes du placement institutionnel ", Lausanne, Cahiers de l'EESP, $\mathrm{n}^{\circ} 15,1993$, $229 \mathrm{p}$.

(8) Pierre Rosat, Chronique des discours et idées qui précédèrent la création d'un établissement pour orphelins à La Chaux-de-Fond, mémoire de licence, FAPSE, université de Genève, 1987 ; Anne Schmutz, L'institut de Drognens. Une maison pour "garçons difficiles", 1889-1963, mémoire de licence présenté à la faculté des lettres de l'université de Fribourg, février 1997, 155 p. ; Sylvie Guinand, L'orphelinat cantonal de Neuchâtel, fondation Borel. Évolution de l'assistance à l'enfance au XIXème et au XXème siècles, mémoire de licence réalisé sous la direction du prof. $\mathrm{Ph}$. Henry, université de Neuchâtel, Institut d'histoire, 1999, $101 \mathrm{p}$.

(9) Voir notamment Le pédagogue et la modernité. À l'occasion du 250ème anniversaire de la naissance de Johann Heinrich Pestalozzi (17461827) : Actes du colloque d'Angers (9-11 pables, est intéressante à plus d'un titre.

Par sa place et sa structure cantonale, la Suisse a joué un rôle certain dans la transmission des modèles d'éducation correctionnelle, mais il semblerait qu'elle en ait aussi produit, reconnus dans les pays voisins comme des modèles ou systèmes typiquement suisses. Trois ont été, en particulier, signalés dans la littérature de l'époque ou dans des congrès internationaux : le modèle de l'école des pauvres, et en particulier celui de Hofwyl dans le canton de Berne, le système pénitentiaire mixte, dit "système de Genève", et le "système par famille" qui a cours dans les établissements d'éducation protestante, fondement de l'éducation philanthropique.

Cette éducation philanthropique, présentée dans les rapports annuels de la colonie agricole de Serixsur-Oron dans le canton de Vaud - le "Mettray suisse romand" - comme une pédagogie par l'amour, contient ces trois modèles et systèmes : l'école des pauvres, le système par famille, l'amendement par le travail et le retour sur soi.

Cet article a pour objectif de donner une place à la Suisse dans l'histoire de l'éducation correctionnelle sous la forme d'un triptyque, "modèles, systèmes et méthodes”, lequel est proposé dans un souci de questionner par ricochet cette histoire des enfants corrigés, enfants protégés, qui forme la genèse de la protection de l'enfance entre 1820 et 1914. Qu'est-ce qui nous unit ? et qu'est-ce qui nous différencie? sont des questions importantes à l'heure où l'historiographie se fait volontiers comparative et européenne. 


\section{L'éducation correctionnelle : une éducation du peuple}

Les maisons de correction, telles qu'elles se développent en Suisse romande dans la seconde moitié du XIXème siècle, sous la forme de colonies agricoles, tiennent à la fois de l'asile des pauvres et de la prison. Alternatives à la prison pour les enfants ayant agi sans discernement, puis pour les enfants en correction paternelle et pour ceux ayant agi avec discernement, elles accueillent aussi, sur le modèle des asiles de relèvement moral, des enfants répondant aux catégories morales d'enfant abandonné moralement, enfant vicieux, enfant indiscipliné et, depuis l'obligation de l'instruction inscrite dans la Constitution helvétique en 1874, enfant " récalcitrant au minimum d'instruction obligatoire ». Mais ces asiles ruraux, qui s'ouvrent dès le début du siècle pour les enfants abandonnés moralement, ont des racines encore plus lointaines dans l'éducation populaire prônée par Johan Heinrich Pestalozzi (1746-1827) et Philippe Emmanuel de Fellenberg (1771-1844).

La figure mythique de Pestalozzi, personnage-culte en Suisse, nommé citoyen d'honneur en France en 1792, et ses diverses expériences d'éducation populaire, en particulier des enfants pauvres, marquent indéniablement, depuis la fin du XVIIIème siècle, l'histoire des institutions et des idées pédagogiques et éducatives en Suisse et dans le monde.(9) Certes les œuvres de Pestalozzi n’ont été que partiellement éditées en français, alors qu'elles l'ont été intégralement en allemand dans l'Édition critique. ${ }^{(10)}$ Néanmoins, un plan de traduction des œuvres en français est actuellement en voie d'exécution, grâce à Michel Soëtard et au Centre de documentation et de recherche Pestalozzi à Yverdon en Suisse romande. Dépassée la difficulté d'accès à la langue de Pestalozzi et à ce que Soëtard décrit comme " un torrent qui dévale d'une montagne d'expériences et charrie tous les styles dans le lit d'un Moi débordant de tensions exacerbées, d'espérances partiellement abouties, de rêves avortés ", ${ }^{(1)}$ le lecteur francophone accédera, dans un proche avenir, à la traduction française des Écrits sur le Neuhof, première expérience du pédagogue suisse, datant de 1768.

Dans ces lettres, écrites entre 1775 et 1778, les ingrédients d'une politique éducative, appelée au siècle suivant éducation correctionnelle, sont juillet 1996), réunis par

Michel Soëtard et

Christian Jamet, Berne,

Peter Lang, collection

Exploration, 1998,

$238 \mathrm{p}$.

(10) Fables de Pestalozzi, choisies et traduites par Jean Moser, Fribourg, éd. Egloff, 1946 ; Lettre de Stans, nouvelle traduction et introduction de Michel Soëtard, Yverdon-les-Bains, Centre de documentation et de recherche, 1985 ; Esquisse de la révolution, traduction et introduction de Philippe Henry, Pierre Marc et Irène Keller, Cabier Vous avez dit... pédagogie ?, Neuchâtel, université, 1991 ; Comment Gertrude instruit ses enfants : un essai pour introduire les mères à l'art d'enseigner elles-mêmes leurs enfants, traduction, introduction et notes de Michel Soëtard, Albeuve (Suisse), Castella, 1985 ; Léonard et Gertrude, 
tomes I et II, traduction de Léon Vassenhove, Neuchâtel, édition de La Baconnière, 1948 ; Chant du cygne, suivi de Mes destinées, traduction de Léon Vassenhove, Neuchâtel, édition de La Baconnière, 1947 ; Mes recherches sur la marche de la nature dans l'évolution du genre bumain, traduction, introduction et commentaires de Michel Soëtard, Lausanne, éditions Payot, 1994.

(11) Michel Soëtard, « La praxis pestalozienne entre poiesis et theoria / Denkmal Pestalozzi, Bildungsforschung und Bildungspraxis ", Éducation et recherche /

Educazione e ricerca, Revue suisse des sciences de l'éducation, Fribourg, Édition universitaire, 1995 , p. $178-186$.

(12) Peter Stadler, «Pestalozzi. Le pédagogue dans l'histoire ", présents : l'éducation du peuple, l'institution éducative comme entreprise rentable, le travail (industriel) des enfants, la formation de citoyens utiles au pays et l'éducation morale et religieuse.

Dans son Appel aux philantbropes et aux bienfaiteurs de l'bumanité en faveur d'un institut d'éducation et de travail pour les enfants pauvres de la campagne, qui date de décembre 1777, Pestalozzi écrit :

"L'expérience m'a montré que ce n'est pas le travail régulier qui freine la croissance et le développement de la jeunesse pauvre et qui nuit à sa santé, mais une existence désordonnée, le manque fréquent du nécessaire, la gloutonnerie quand l'occasion s'en présente, et plus encore les passions effrénées, la sauvagerie, l'agitation constante, l'irritation et l'abattement. " Pour le pédagogue, l'oisiveté, la mendicité et la passion de mal faire sont à combattre par un travail régulier. Le philanthrope qu'est Pestalozzi veut sauver en éduquant. Sauver à la fois l'âme et la moralité de l'enfant, le pays et l'humanité.

L'influence de Pestalozzi en France reste à écrire. Il semblerait, selon Peter Stadler, ${ }^{(12)}$ que la France officielle, après la Révolution, n'ait guère fait de cas de sa méthode et que les lycées furent organisés d'après le modèle militaire. Mais l'influence est peut-être parvenue à travers un autre modèle, celui de l'école des pauvres de Fellenberg et de la Rauhe Haus d'Emmanuel Wichern de Hambourg qui s'en inspirent directement, dont il s'agirait aussi de mieux étudier l'influence en France.

\section{L'école des pauvres : un modèle international}

En 1879, le médecin et philanthrope neuchâtelois Paul-Louis Ladame effectue un voyage à travers la Suisse et l'Europe pour y étudier les orphelinats. Dans l'ouvrage qui en rend compte, il présente l'établissement de Hofwyl, près de Berne, fondé par Fellenberg, comme « le point de départ de toutes les colonies agricoles ". ${ }^{(13)} \mathrm{Il}$ ajoute :

«La France, l'Allemagne, la Hollande, l'Angleterre prirent modèle sur cet institut célèbre et presque tous les pays d'Europe et de l'Amérique du Nord possèdent maintenant des institutions agricoles, colonies où les enfants sont répartis en groupes ou familles cherchant à imiter le plus possible la vraie famille, le vrai foyer domestique. ${ }^{(14)}$ 
Pour Ladame, le système de groupe ou de famille qui régit la colonie de Mettray en France remonte à la fondation de Fellenberg, inspirée de Pestalozzi. Il apparaît évident pour le médecin que le modèle de Mettray, imité de la Rauhe Haus, est en fait celui de Hofwyl qui a transité par Hambourg.

Fellenberg est, aujourd'hui, moins connu que Pestalozzi, bien qu'il ait aussi œuvré pour l'éducation du peuple, notamment dans le Conseil de l'éducation sous la République helvétique. ${ }^{(15)}$ Pourtant, certains travaux en langue française ${ }^{(16)}$ cherchent à montrer le rôle essentiel de ce philanthrope bernois, alors de renommée européenne, comme agronome et comme pédagogue.

Certes, la République pédagogique qu'il crée à Hofwyl, dans le canton de Berne, tient du Neuhof de Pestalozzi, qui n'est alors qu'à quelques kilomètres, mais elle se réfère aussi au Philanthropinum de Johan Bernard Basedow (1723-1790), avec cette idée d'un côtoiement des classes sociales, des âges et des fortunes, dans un cadre pédagogique orienté vers l'apprentissage, par l'expérience, des différences sociales : intérêt du riche pour le pauvre, travail du pauvre et reconnaissance à l'égard du riche. À Hofwyl, entre 1799 et 1841, sont réunis sur une même terre un institut agricole scientifique, une école de pauvres, une école normale, une institution pour fils de familles de haut rang, une colonie agricole, un collège d'enseignement général [Realschule], une école pour jeunes filles pauvres et une école de soins aux petits enfants, qui forment un état de l'éducation [Erziebungstaat], un état idéal, une utopie en quelque sorte.

Mais c'est surtout l'école des pauvres qui aura une renommée mondiale, grâce à son instituteur Johan Jakob Werhli (1790-1855), grâce aux visiteurs, grâce aussi aux rapports des étrangers venus, en pèlerins, visiter l'œuvre de "l'agriculteur philosophe ", du " génie du siècle ", de " l'apôtre de l'agriculture ", et grâce encore aux articles parus dans La bibliothèque britannique, qui se charge, entre 1808 et 1830 , de faire connaître l'œuvre au public francophone, notamment sous la plume du Genevois Charles Pictet-de-Rochemont. Outre les correspondances de Fellenberg avec certains Français, par exemple Charles Fourier, ${ }^{(17)}$ il est in Le pédagogue et la modernité..., op. cit., p. $37-50$.

(13) Dr. Paul-Louis

Ladame, Les orphelinats de la Suisse et des principaux pays de l'Europe. Avec un aperçu historique, Paris, Neuchâtel, Genève, 1879.

(14) Ibid., p. VI-VII.

(15) En 1794, il se rend à Paris où il est secrétaire de la légation de la République helvétique.

(16) Voir Marc

Vuilleumier, « Ph. E. de Fellenberg, Fourier et l'école sociétaire ", Cabier Charles Fourier, $n^{\circ}$ 6, 1995, p. 15-27 ; André Brandt,

"Philippe-Emmanuel de Fellenberg et ses relations avec l'Alsace ", Bulletin du musée historique de Mulhouse, 1968, p. 155-197 ; Martine Ruchat, "Pédagogie de la conscience. De l'école 
des pauvres de Hofwyl

à la colonie agricole de

Serix-sur-Oron en Suisse

protestante ", Société

et représentation, "Michel

Foucault, Surveiller et

punir : la prison vingt

ans après", CREDHESS,

$\mathrm{n}^{\circ} 3$, novembre 1996 ,

p. $269-276$.

(17) Voir Marc Vuil-

leumier, op. cit., p. 15-27.

(18) Édouard

Ducpétiaux, Colonies

agricoles, écoles rurales et

écoles de réforme pour

les indigents, les mendiants

et les vagabonds et spé-

cialement pour les enfants

des deux sexes en Suisse,

etc., Bruxelles, imprime-

rie Lesigne, 1851.

(19) Gabriel de Lurieu

et H. Romand, Études

sur les colonies agricoles

de mendiants, jeunes déte-

nus, orphelins et enfants

trouvés. Hollande-Suisse-

Belgique-France, Paris,

Librairie agricole de la

Maison rustique, 1851.
(20) André Brandt,

«Philippe-Emmanuel de

Fellenberg et ses rela-

tions avec l'Alsace ",

Bulletin du musée histo-

rique de Mulhouse, 1968.

(21) Ibid.

(22) Voir Martine

Ruchat, Les chroniques

du mal. Une histoire de

l'éducation correctionnelle

au 19ème siècle, thèse

$n^{\circ} 170$, faculté de Psy-

chologie et des Sciences

de l'éducation, 1990. indéniable que Pictet-de-Rochemont servira de relais entre le canton de Berne et la France, transitant par Genève et Paris, notamment par ses liens avec la Société d'agriculture de la Seine et son président François de Neufchâteau. Par ailleurs, les rapports de visiteurs comme Édouard Ducpétiaux ${ }^{(18)}$ ou Gabriel de Lurieu et H. Romand, ${ }^{(19)}$ citent Hofwyl. En 1829, c'est au tour du baron Joseph-Marie de Gérando de se faire propagandiste de l'institut après y avoir passé deux jours. ${ }^{(20)}$ De nombreux liens s'établissent aussi entre Hofwyl et l'Alsace, puisque certains fabricants alsaciens y envoient leurs enfants, notamment Jacques Gros de la manufacture de Wesserling. ${ }^{(21)}$ Sans oublier aussi que Goethe se servira du modèle d'Hofwyl pour décrire la Province pédagogique dans Les années du voyage de Wilhelm Meister.

L'école des pauvres d'Hofwyl offre une éducation centrée sur le travail agricole, auquel s'ajoutent les travaux dits industriels liés à l'agriculture : charronnage, menuiserie, ferronnerie, tressage de la paille. Cette éducation au travail vise deux objectifs essentiels : donner une formation aux pauvres sans les arracher à leur état et, par leur travail, couvrir les frais de l'établissement. L'intérêt de Pictet-de-Rochemont pour l'agronomie et l'éducation le dirige vers l'œuvre de Fellenberg, qu'il découvre en 1807. En 1816, il le consulte pour un projet d'ouverture, à Genève, d'une école rurale qui reprendrait les principes et l'esprit d'Hofwyl, où un jeune homme, Jacob, dit Jean-Jacques Eberhardt, est envoyé se former aux objectifs de travail, ordre et paix. ${ }^{(22)}$ 


\section{Le système mixte, dit "système de Genève"}

Les maisons de correction relèvent aussi des réformes pénale et pénitentiaire qui visent, dès 1810, un traitement spécifique pour les enfants. En effet, le nouveau code pénal napoléonien, en Suisse comme en France, ${ }^{(23)}$ introduit une limite d'âge, seize ans, à l'indulgence de la loi et une distinction, à la fois morale et psychologique, entre les enfants conscients ou non du caractère délictueux de l'acte au moment où il est commis et de la rigueur de la peine encourue. À ces deux catégories d'enfants correspondent des traitements particuliers : les enfants "discernants" recevront, en prison, une peine correctionnelle visant une rectification de l'éducation (art. 67), les enfants "non-discernants" iront en maison de correction (art. 66) au maximum jusqu'à l'âge de vingt ans. Néanmoins, à Genève, les deux catégories se retrouveront en prison faute de maison de correction, ceci jusqu'à l'ouverture, en 1863, de la colonie agricole de Serix-sur-Oron.

Pendant ces années 1810-1860, à Genève, la réforme pénitentiaire bat son plein. D’abord emprisonnés dans la prison d'Ancien Régime dite "Ancien Évêché", dans laquelle toutes les catégories de détenus sont mélangées, les enfants seront, dès 1825, dans un souci de protection, détenus dans la prison pénitentiaire construite selon le modèle panoptique (semi-panoptique) de Jeremy Bentham (1748-1832). ${ }^{(24)}$ Les enfants en correction paternelle et ceux condamnés jusqu'à trois mois resteront dans l'ancienne prison, alors que les condamnés à des peines de plus de trois mois seront enfermés dans la nouvelle prison dans un quartier dit d'exception. Ce quartier d'exception, appelé, dès 1833, “quartier des jeunes et des améliorés", regroupe les enfants de moins de seize ans, exceptionnellement les seize/dix-huit ans et les détenus qui le méritent. Car, outre l'introduction du travail comme fondement du régime et la solitude pendant la nuit (système auburnien), l'amendement dépend d'une modulation des peines en fonction de la bonne ou de la mauvaise conduite, en quelque sorte d'un système d'intimidation.

Entre 1840 et 1843, l'ancienne prison de détention de l'Évêché est entièrement reconstruite. Elle permettra d'accueillir les détenus selon le principe de l'encellulement total nuit et jour (système philadelphien). L'isole-
(23) L'occupation française s'étendra de 1798 à 1814 .

(24) Robert Roth, Pratiques pénitentiaires, théories sociales. L'exemple de la prison de Genève, 1825-1862, Genève, librairie Droz, 1981. 
(25) Compte-rendu $d u$ congrès de Francfort-surle-Main, 28, 29 et 30 septembre 1846, Paris, imprimerie de E. MarcAurel, 1847, 40. ment est présenté comme un régime de faveur pour les présumés innocents, les délinquants légers et surtout pour les enfants, toujours dans ce souci de protection. L'existence de deux prisons, de détention et pénitentiaire, permettra à Genève de développer un double système, ou système aménagé, ou encore système progressif par classifications, présenté en 1846, au congrès pénitentiaire de Francfort-sur-le-Main, comme le "système de Genève".

En quoi consiste l'aménagement ? En un isolement complet de tous les prévenus pendant l'instruction et en un isolement absolu pour les condamnations de courte durée. Quant aux condamnés à des peines de plus longue durée, ils sont placés en isolement en début de peine, lequel sera modulé en fonction du comportement du détenu. Ainsi, à la prison pénitentiaire, le système auburnien se teinte en quelque sorte de système philadelphien. L'enfant de moins de seize ans, par exemple, est soumis à quinze jours de détention solitaire et silencieuse, dont cinq sans occupation aucune, si ce n'est la lecture de livres saints. À la prison de détention de l'Évêché, le système philadelphien est lui aussi aménagé, puisque les détenus travaillent en commun, avec promenade en file indienne. Ce système dit "de Genève" permet aussi des modulations de traitement par un jeu d'atténuation ou d'accentuation de l'isolement considéré comme un moyen de compression des passions et de connaissance de soi. Au congrès pénitentiaire, le chapelain des prisons de Genève, Ferrière, en fait l'apologie :

«[...] l'homme en cellule avoue mieux, ouvre mieux son cœur, reste mieux sous l'impression d'effroi et de conscience qu'on a gravé en lui ; il comprend aussi mieux quel est son intérêt bien entendu terrestre ; il a davantage le sentiment de la présence de Dieu. " ${ }^{(25)}$

Ce système progressif sera essentiel aussi à l'éducation correctionnelle.

Dès 1848, sous la poussée du régime radical, le système philadelphien sera assoupli : à la prison de détention, les détenus sont réunis dans des dortoirs. Quant à ceux qui sont soumis au régime cellulaire, ils doivent être visités au moins huit fois par jour. Le nouveau règlement des prisons genevoises de 1867 ne sera modifié qu'en 1909. 


\section{Le système par famille : un "grand succès suisse"}

Dans l'éducation philanthropique des asiles de relèvement et dans l'éducation correctionnelle des colonies, en Suisse protestante, le "système par famille" est présenté comme le moyen le plus naturel de ramener l'enfant au bien. Offrir aux enfants, par l'expérience et pour nourrir les sentiments filiaux, un modèle qui a manqué ou qui était, de l'avis des philanthropes, un "mauvais exemple" est un moyen éducatif essentiel qui complète celui non moins essentiel du travail. Au congrès pénitentiaire de Bruxelles, en 1900, le système par famille est présenté comme un "grand succès suisse".

D'ailleurs, travail et famille sont intimement liés. La "famille" est un groupe d'une douzaine d'enfants qui partagent l'habitat, le dortoir notamment, et le travail. Certes, à Mettray par exemple, la famille comprendra des groupes beaucoup plus nombreux. Henri Joly, qui, en 1902, part à la recherche de l'éducation correctionnelle à travers l'Europe, ${ }^{(26)}$ relèvera du côté protestant cette particularité du petit groupe, qu'il expliquerait par de plus grandes ressources financières. ${ }^{(27)}$ Mais le petit groupe a aussi une fonction éducative et disciplinaire. Wehrli, à Hofwyl, décrit très bien comment, à la tête de sa famille, par une pédagogie naturaliste, il enseigne tout en travaillant :

« En glanant sur la même ligne, nous nous tenions à une certaine distance les uns des autres, mais pas trop loin cependant, soit pour ne pas laisser les épis, soit pour ne pas perdre l'instruction que je leur donnois tout en travaillant : j'avois soin, pour cela, de me tenir au milieu du rang. Tous les objets qui tomboient sous les sens des enfans étoient des occasions de conversations. L'un par exemple, découvroit un insecte inconnu et m'en demandoit le nom. D'autres faisoient la même chose pour des plantes ou pour des pierres. J'en prenois occasion d'instruire tous les autres. Comme toutes les questions s'adressoient à moi, tous vouloient travailler à mes côtés pour bien comprendre ce que je disois, et c'étoit à qui se rapprochoient le plus de moi. Cependant il ne falloit pas que le travail en souffrit, et que la conversation, au lieu d'être l'accessoire, devint le principal. Voici à quel expédient j'eu recours : lorsqu'un de mes élèves qui se trouvoit près de moi se laissoit détour-
(26) Henri Joly, À la recherche de l'éducation correctionnelle à travers l'Europe, Paris, librairie Victor Lecoffre, 1902.

(27) Peter Chmelik constate que $80 \%$ des établissements en Suisse allemande protestante reçoivent, au XIXème siècle, de 20 à 55 enfants. Seuls $6 \%$, soit 4 homes, en reçoivent plus de 60. Pour la Suisse romande, Serixsur-Oron a un potentiel de 65 lits ; seuls

Drognens et la SainteFamille de Sonnenwyl dans le canton de Fribourg, ainsi que la Montagne-de-Diesse (Jura bernois), reçoivent plus de 100 enfants.

Cité par Anne Schmutz, op. cit., p. 65. 
(28) Le « journal de Werhli » est une collection des lettres de ce personnage à ses parents, dans lesquelles il rend compte de la marche de son école. Le rapporteur y puise avec abondance faits et observations.

«Rapport sur l'institut des pauvres de Hofwyl, rédigé par Mr. A. Rengger, ci-devant ministre de l'Intérieur de la République helvétique et au nom de la Commission établie pour l'inspection de l'établissement ", Berne, 1814, in Bibliothèque Britannique, t. 56, 1814, p. $482-485$.

(29) Martine Ruchat, «Pédagogie de la conscience... ", op. cit. ner du travail par la conversation, je l'envoyois au bout du rang. Cette petite punition suffit à maintenir leur attention au travail du glanage. " (28)

Dans l'école rurale de Carra, à Genève, ouverte par Pictet-de-Rochemont sur le modèle d'Hofwyl, les enfants seront organisés en "bandes", avec une surveillance exercée par un élève choisi par le maître. Cette organisation assure l'émulation entre les enfants, renforcée par un système de bonnes notes convertibles en argent. Car, si, selon les dires des philanthropes, il faut faire le plus de bien possible avec peu d'argent, c'est aussi par les privations et les avantages des profits matériels que les enfants apprendront ce qu'est le bonheur de travailler.

La famille n'est pas seulement une source d'affections utiles à la régénération morale, elle est un modèle social, économique et politique. Elle devient même la base de l'économie, par le travail de ses membres. Mais elle est aussi conçue comme un modèle de société qui renvoie au modèle national : « la société est une grande famille, la famille est une petite société ». L'éducation dans la maison de correction de Serix-sur-Oron vise à faire des "citoyens utiles au pays ", à savoir des travailleurs qui participeront à l'économie nationale (notamment par l'agriculture), et qui, de ce fait, ne seront pas une charge pour la société. La responsabilité individuelle d'un potager devient le lieu d'apprentissage naturel, intuitif, du principe du " qui veut vivre doit travailler », du " sans effort aucun fruit ", du " sans travail aucune propriété ", cher déjà à Fellenberg et à Werhli. Le travail par famille se double alors d'un travail individuel, formateur à la fois à la prévoyance, au sentiment de propriété et à la discipline, mais aussi d'un travail de conversion du mal en bien. ${ }^{(29)}$

\section{La pédagogie par l'amour}

La pédagogie par l'amour prônée par les philanthropes est d'abord un développement des affections domestiques, lequel doit s'effectuer intuitivement, naturellement, dans le petit groupe, c'est-à-dire la famille. Un enfant qui serait fermé à ce sentiment d'affection pour le père et la mère, ou pour le sous-maître, est un enfant perdu pour l'éducation correctionnelle. C'est aussi une pédagogie par l'exemple donné par le frère aîné ou le sous-maître qui dirige la famille, ou encore par les anciens élèves repentis, dont on relate la réussite. L'enfant doit être gagné par le cœur. 
L'amour ne va pas sans deux autres affections, celle du respect de l'autorité et donc celle de l'obéissance. Aimer, c'est obéir ; désobéir, c'est faire du chagrin au maître. Dans cette dynamique culpabilisante, l'enfant n'a que deux solutions. Soit il avoue, regrette et pleure, signe de sa repentance qui entraîne le pardon, soit il résiste, s'enferme dans l'orgueil, signe du vice qui, de l'avis des philanthropes, appelle des privations de nourriture, de sorties, de liberté, à savoir le cachot. Celui-ci devient alors un ultime espoir d'amendement suscité par le recueillement. Sinon, il reste le renvoi de l'institution pour la prison correctionnelle. Encore un système progressif comme levier d'intimidation.

C'est alors que l'éducation correctionnelle rejoint la tradition pénitentiaire, pour ne pas dire pénitencielle, du silence, du recueillement, du retour sur soi, encouragés si nécessaire par la cure d'âme. À la colonie de Serix-sur-Oron, le retour sur soi est d'abord suscité par une surveillance omniprésente du maître ou du sous-maittre, qui oblige à un contrôle de soi-même. Tout écart entraîne un rappel à l'ordre qui en appelle, certes, aux punitions, mais surtout à la conscience individuelle : avouer sa faute, s'en repentir, promettre de ne plus recommencer.

Si le comité de Serix rappelle continuellement qu'une pédagogie par l'amour doit être exempte de corrections corporelles, celles-ci peuvent exceptionnellement être données, mais seulement par le directeur. Par contre, à Drognens dans le canton catholique de Fribourg, à la colonie de Saint-Nicolas ouverte en 1889 et dirigée par la Société du Divin Sauveur, la correction apparaît comme " utile et nécessaire ", mais à infliger avec prudence. ${ }^{(30)}$ Selon l'Étude sur les principes éducatifs généraux sur lesquels doivent reposer le Règlement et la Direction de l'Institut de Drognens, datant de $1915,{ }^{(31)}$ il s'agit de donner aux garçons indisciplinés et coupables de délits une triple formation : morale, intellectuelle et physique. Alors que la pédagogie protestante se porte garante de respecter chez l'enfant la vocation divine en évitant à tout prix un "déclassement par le haut", les principes éducatifs établis par les Salvatoriens voient les études supérieures en vue de diplômes ou de brevets comme souhaitables pour les enfants pauvres.
(30) Anne Schmutz, op. cit.

(31) Ces "principes" sont rédigés par E. Bise, doyen, membre de la commission d'éducation, Vuisternens-enOgoz, le 23 juillet 1915. 
(32) « Des philanthropes aux politiques sociales (XVIIIèmeXXème) ", Cabier de l'Association pour la recherche sur les philanthropies et les politiques sociales, 1, 1992.
Mais mises à part ces différences, à Drognens comme à Serix, on dote l'enfant d'un carnet d'épargne pour le former à la prévoyance, comme on le dote d'une famille, dont le directeur sera le père, et d'un travail présenté non seulement comme un grand moralisateur ( $c f$. Pestalozzi), mais comme un grand redresseur.

\section{Conclusion}

En conclusion, le champ correctionnel qui se développe au XIXème siècle apparaît comme un réseau européen - on a pu parler de l'internationale philanthropique ${ }^{(32)}$ - constitué de congrès, de visites, de rapports sur les établissements, de correspondances. Dans ce réseau de sociabilité, la Suisse a indéniablement sa place, qu'une histoire comparée permet de mieux comprendre. Mais c'est aussi par l'histoire des mentalités que la spécificité helvétique pourrait être plus finement approchée : la famille comme modèle politique, le rôle de l'argent dans l'éducation morale, la pédagogie protestante centrée sur la conscience individuelle, l'usage d'un système progressif comme forme d'exercice du pouvoir. Et, dans cette perspective, les pleurs comme évidence de la soumission renverraient à la fois aux affections domestiques du système par famille, au processus d'intimidation entraîné par le système progressif d'amendement, à une éducation du cœur chère à Pestalozzi.

Mais pleurait-on plus à Serix qu'à Mettray, ou dans le Mettray hollandais dont parle Jeroen Dekker? 